\title{
Yoga versus education for Veterans with chronic low back pain: study protocol for a randomized controlled trial
}

\author{
Robert B. Saper ${ }^{1 *}$, Chelsey M. Lemaster ${ }^{1}$, A. Rani Elwy ${ }^{2,3}$, Ruth Paris ${ }^{4}$, Patricia M. Herman ${ }^{5}$, Dorothy N. Plumb ${ }^{6}$,
} Karen J. Sherman ${ }^{7,8}$, Erik J. Groess ${ }^{9,10}$, Susan Lynch ${ }^{11}$, Shihwe Wang ${ }^{6}$ and Janice Weinberg ${ }^{12}$

\begin{abstract}
Background: Chronic low back pain is the most frequent pain condition in Veterans and causes substantial suffering, decreased functional capacity, and lower quality of life. Symptoms of post-traumatic stress, depression, and mild traumatic brain injury are highly prevalent in Veterans with back pain. Yoga for low back pain has been demonstrated to be effective for civilians in randomized controlled trials. However, it is unknown if results from previously published trials generalize to military populations.

Methods/design: This study is a parallel randomized controlled trial comparing yoga to education for 120 Veterans with chronic low back pain. Participants are Veterans $\geq 18$ years old with low back pain present on at least half the days in the past six months and a self-reported average pain intensity in the previous week of $\geq 4$ on a $0-10$ scale. The 24-week study has an initial 12-week intervention period, where participants are randomized equally into (1) a standardized weekly group yoga class with home practice or (2) education delivered with a self-care book. Primary outcome measures are change at 12 weeks in low back pain intensity measured by the Defense and Veterans Pain Rating Scale (0-10) and back-related function using the 23-point Roland Morris Disability Questionnaire. In the subsequent 12-week follow-up period, yoga participants are encouraged to continue home yoga practice and education participants continue following recommendations from the book. Qualitative interviews with Veterans in the yoga group and their partners explore the impact of chronic low back pain and yoga on family relationships. We also assess cost-effectiveness from three perspectives: the Veteran, the Veterans Health Administration, and society using electronic medical records, self-reported cost data, and study records.
\end{abstract}

Discussion: This study will help determine if yoga can become an effective treatment for Veterans with chronic low back pain and psychological comorbidities.

Trial Registration: ClinicalTrials.gov: NCT02224183

Keywords: Veterans, Low back pain, Military families, Randomized controlled trial, Yoga, Cost-effectiveness

\section{Background}

\section{Low back pain in Veterans}

Musculoskeletal pain conditions are the most commonly diagnosed medical problems among the more than two million Veterans from Operations Enduring Freedom, Iraqi Freedom, and New Dawn (OEF/OIF/OND), far surpassing other medical and mental health disorders

\footnotetext{
* Correspondence: robert.saper@bmc.org

'Department of Family Medicine, Boston University School of Medicine and Boston Medical Center, Boston, MA, USA

Full list of author information is available at the end of the article
}

[1-3]. In a study of 91,000 Veterans receiving care from the Veterans Health Administration (VHA), 43 \% reported having any pain, and $63 \%$ of these reported moderate to severe pain [4]. Eighty percent of VHA visits include pain-related complaints [5]. Overreliance on opioids is common and can lead to adverse effects ranging from sedation to dependence, addiction, and death due to overdose $[6,7]$. Among pain conditions, chronic low back pain (cLBP) is the most frequent [4] and causes substantial suffering, decreased functional capacity [8], and lower quality of life $[9,10]$. The direct costs of 
chronic pain in the U.S. are estimated to be $\$ 100$ billion or greater annually [3]. Among Veterans, back pain is a leading cause of disability $[11,12]$.

Psychological distress, back pain, and disability are strongly correlated. Symptoms of post-traumatic stress [13], depression [6], and traumatic brain injury [1] are highly prevalent in Veterans with cLBP [14]. Among Veterans receiving care from VHA Polytrauma Network sites, $42 \%$ had the triad of chronic pain, post-traumatic stress symptoms (PTSS), and persistent post-concussive symptoms (traumatic brain injury) [7, 15]. Low pain selfefficacy and maladaptive pain coping behaviors (e.g., catastrophizing [16], fear avoidance [17], and substance abuse [18]) compound back pain recovery. Moreover, stigma and other barriers often prevent Veterans from seeking mental health care (e.g., cognitive therapy for PTSS), leading to additional barriers to back pain recovery $[19,20]$.

According to the Institute of Medicine, "Military family members are an important part of the readiness and well-being of the military force." [21] Several studies on the impact of chronic pain on family life in civilians have shown poorer couple agreement [22] and lower marital satisfaction [23]. Injured service members and their partners must adapt to the injury's physical and emotional sequelae, including chronic pain, post-traumatic stress disorder (PTSD), and depression [24, 25]. Partners of injured Veterans experience elevated distress levels along with the greater burden of caregiving for the Veteran, household, and children [26, 27]. Although chronic pain in Veterans is a significant and growing problem [2], its impact on Veteran families has rarely been studied.

\section{Yoga for low back pain}

Yoga is increasingly common with more than $8 \%$ of U.S. adults reporting use in 2012 [28-31]. A 2005 randomized controlled trial (RCT) found a moderate benefit of yoga for improving back-related function in civilian adults with cLBP [32]. This prompted the VHA, American College of Physicians, and American Pain Society to list yoga in clinical practice guidelines as an evidencebased treatment for cLBP [33]. Subsequently, four moderate to large RCTs $(n=90-313)$ [34-37] and six smaller $(n=20-60)$ [38-43] RCTs have also shown yoga to be effective for reducing pain and improving function in civilian adults with cLBP. Meta-analyses support these conclusions [44-46]. Several yoga-cLBP studies found associated psychological benefits in mood and selfefficacy [34, 36, 42, 43, 47]. Yoga research on psychological health is growing, showing promising evidence for benefit in depression [48-56], anxiety [56-59], and insomnia [60, 61]. Yoga classes can also increase social connectedness and spirituality [62].
The 2010 Army Surgeon General Pain Management Task Force Report highlighted that pain is an enormous problem facing Veterans and military families [63]. The report emphasized the importance of partnerships to develop an "integrative and interdisciplinary approach" to pain management, including incorporating "integrative and alternative therapeutic modalities into a patientcentered plan of care" [63]. The report emphasized, "Pain cannot be managed without addressing its relationship to stress," and shifting from passive to more active treatments "improves the outcome, decreases provider dependent care, and empowers the patient with a sense of control over his or her condition." The Task Force identified yoga as one of several "Tier 1" complementary modalities as priorities for DoD-VA research and possible integration. A recent VHA report mapping the evidence for yoga on high-impact conditions supported the potential benefit for adults with cLBP [46]. Yoga is increasingly offered to military personnel and Veterans by nonprofit yoga organizations and yoga studios. Among VHA PTSDspecific programs, $29 \%$ offer yoga [64].

Whether results from previously published civilian yoga-cLBP trials generalize to Veteran populations is unknown. In contrast to participants in civilian studies, Veterans with cLBP are more likely to be men with different mechanisms of injury (e.g., direct combat-related trauma or severe non-combat back loading from carrying heavy gear), greater pain severity and disability, and more serious comorbid psychological symptoms [1, 4, $13,15]$. Only two small reports of yoga for cLBP in Veteran settings have been published $[65,66]$. These uncontrolled yoga-cLBP clinical programs in Veterans showed promising improvements in pain and depression symptoms. Despite enthusiasm for offering yoga to Veterans, yoga instruction to date in the VHA is not well standardized or widely implemented. More importantly, there is little strong evidence for yoga's effect on PTSS, depression, or other psychosocial problems. Thus, structured and reproducible yoga protocols for cLBP need to first be adapted to the unique needs of Veterans [3] and then must be rigorously tested for clinical and costeffectiveness in this population.

\section{Specific aims}

This study will determine if yoga can become a safe, clinically effective, cost-effective, and scalable nonpharmacologic approach to address the physical and psychosocial dimensions of cLBP in Veterans [67]. This study is a 24-week two-arm randomized controlled trial for 120 Veterans with cLBP with four specific aims:

1. Primary aim - to determine the effectiveness of a structured, reproducible 12-week series of hatha yoga classes, supplemented with home practice, 
compared to an education control group, for decreasing pain intensity and improving back-related function

2. Secondary aim - to evaluate the effectiveness of yoga compared to education for improving PTSS and other psychosocial outcomes, including depression, anxiety, self-efficacy, and coping

3. Secondary aim - to evaluate the cost-effectiveness of yoga for cLBP at 12 weeks and 24 weeks from three perspectives: the Veteran, the Veterans Health Administration, and society

4. Exploratory aim - to explore the effect of cLBP on family relationships, as well as yoga's impact on the Veteran, family, and family functioning

The results of this study have the potential to impact the approach and management of cLBP according to the Army Surgeon General Pain Management Task Force vision, i.e., having a more integrative and interdisciplinary focus on active self-care approaches that empower Veterans to have greater control of their condition [63].

\section{Methods/design}

\section{Study design}

The trial is a single-blinded 24-week RCT and is divided into two phases: an initial 12-week intervention period followed by a 12-week follow-up period (Fig. 1). Prior to beginning the intervention, Veterans are randomized in a 1:1 ratio into (1) a 12-week series of structured weekly hatha yoga classes supplemented by home yoga practice or (2) education using a comprehensive back pain selfmanagement book, The Back Pain Helpbook, supplemented by newsletters that highlight key content [68]. Block-stratified randomization is being used to ensure similar numbers of Veterans in both treatment groups who are $\leq 45$ years old, experience PTSS, and have longterm partners (spouse, live-in partner, or in a live-out committed relationship). During the 12-week follow-up period, yoga participants are encouraged to continue home practice. Those in the education group are encouraged to continue following recommendations from the book.

The study co-primary endpoints are average pain intensity over the previous 7 days measured by the Defense and Veterans Pain Rating Scale (DVPRS; Fig. 2) $[69,70]$ and back-related function measured by the Roland Morris Disability Questionnaire (RMDQ; scores range from $0-23$ with higher scores reflecting poorer function) [71, 72]. We hypothesize that (1) 12 weeks of weekly yoga will be more effective than education for improving low back pain intensity and back-related function and (2) yoga will be superior compared to education for reducing PTSS in the subset of participants with comorbid PTSS at baseline.
Secondary outcomes include pain medication use, health-related quality of life, and a range of psychological measures targeting key mental health symptoms facing Veterans. Other secondary outcomes include the minimal dataset recommended by the Report of the NIH Task Force on Research Standards for Chronic Low Back Pain [73]. Cost-effectiveness analyses will be performed from the perspective of the Veteran, VHA, and society. To gain a greater understanding of the impact of cLBP on the Veteran and family, we are conducting in-depth interviews with a subset of 20 Veterans in the yoga arm and their partners before and after the yoga intervention.

The Boston University Medical Campus and Bedford VA Institutional Review Boards (IRBs) approved this study.

\section{Sample size}

We assume a two-sided $\alpha=0.05$. This is justifiable because, although there are two primary outcomes, they are not independent. We use 2.5 as the standard deviation for the mean pain change score between baseline and 12 weeks, which is what was found in previous yoga-cLBP studies [37]. We also assume a $20 \%$ dropout rate based on previous studies [37]. Thus, a sample size of 120 participants randomized to yoga vs. education provides $97 \%$ power to demonstrate a minimal clinically significant difference in pain score of 2.0 [74]. For the RMDQ change score, if we assume a standard deviation of $5.0[35,37]$ and a minimal clinically significant difference of 3.0 [75], we have $82 \%$ power to show a statistically significant difference between the two groups. Finally, if we assume that $50 \%$ of the study participants with cLBP have a baseline PTSD CheckList - Civilian Version (PCL-C) score $\geq 30$ and a standard deviation for the PCL-C change score of 12 [76, 77], there is $80 \%$ power to demonstrate a minimal clinically meaningful difference in PCL-C of 10 points.

\section{Inclusion and exclusion criteria}

Table 1 lists the eligibility criteria and the corresponding rationale. Inclusion criteria are: $\geq 18$ years old; low back pain present on at least half the days in the past six months; average back pain intensity $\geq 4$ for the previous seven days on a $0-10$ numerical rating scale; and Veteran of the U.S. military. Exclusion criteria include: back pain caused by inflammatory conditions (e.g., ankylosing spondylitis), malignancy, fracture, or infection; practiced yoga regularly (more than twice per month) or read The Back Pain Helpbook [68] within the past six months; new back pain therapies started in the last month or planned to begin in the next three months; plans to leave the area in the next six months; progressive or severe neurological deficits; lack of consent; 


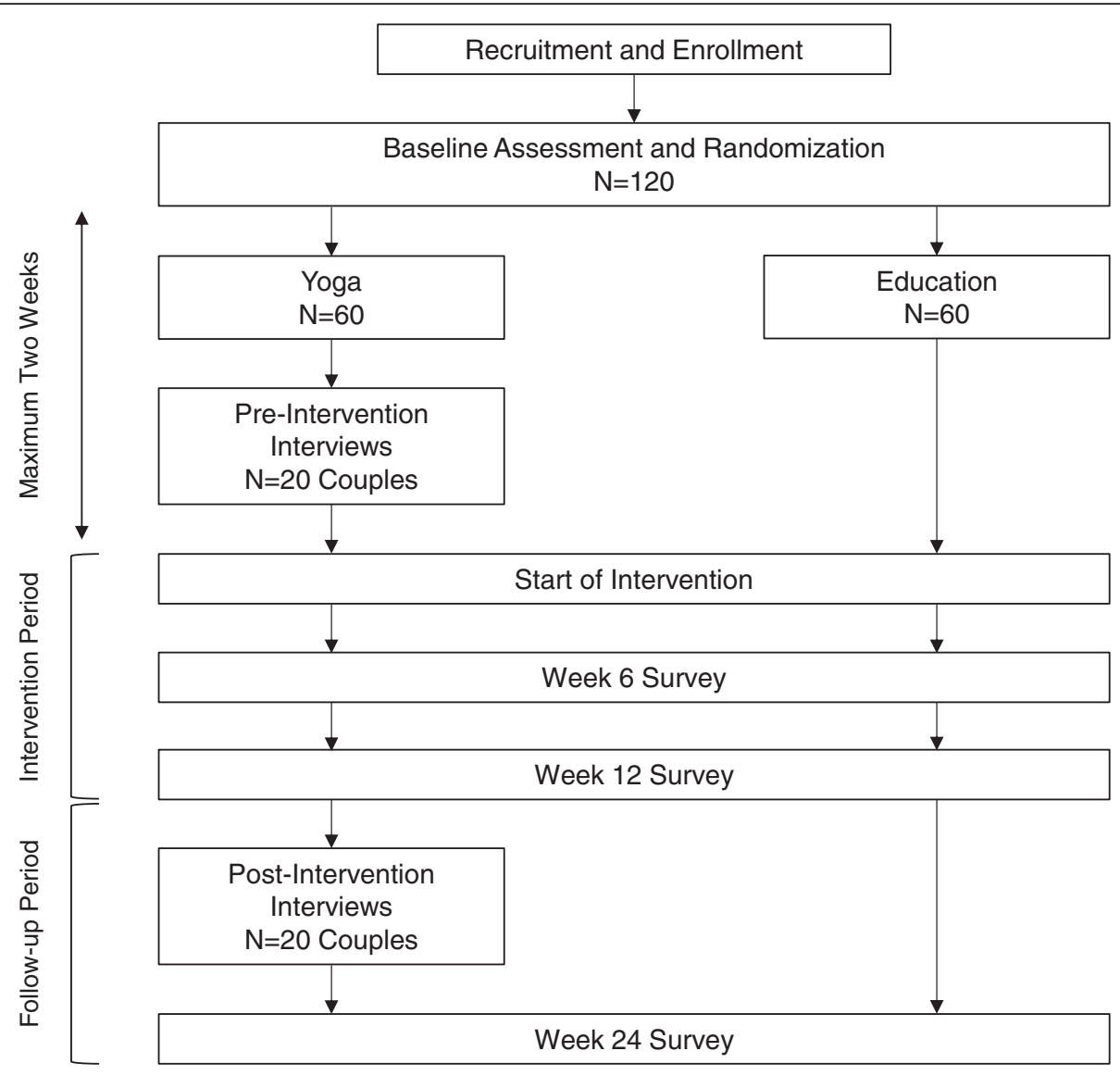

Fig. 1 Study flow diagram. The study will enroll and randomize 120 Veterans equally into yoga and education groups. Qualitative interviews will take place with 20 Veterans in the yoga arm and their partners before and after the 12-week intervention period. Interventions are followed by a 12-week follow-up period. Data collection takes place at baseline (prior to randomization), 6 weeks, 12 weeks, and 24 weeks

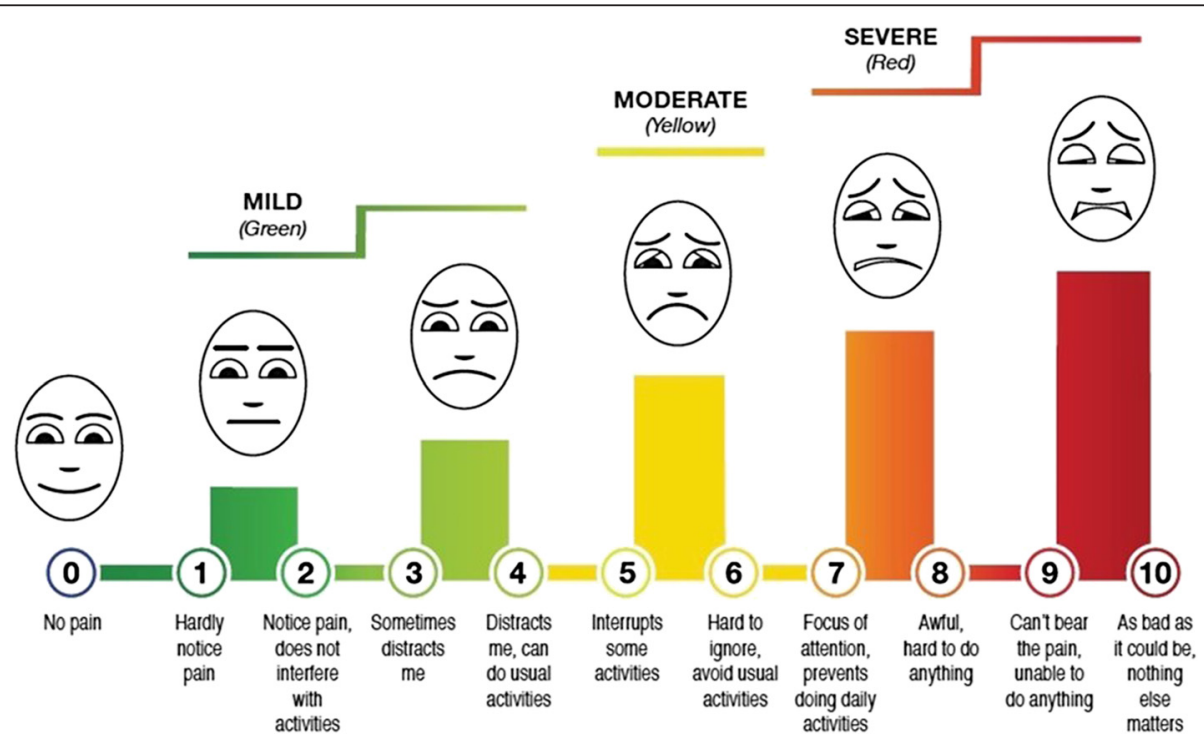

Fig. 2 Defense and Veterans Pain Rating Scale. The Defense and Veterans Pain Rating Scale (DVPRS), developed by the Army Surgeon General Pain Management Task Force, is a validated patient-reported pain assessment tool [69, 70]. This integrated graphic tool incorporates a numerical rating scale, descriptors for each numerical rating, "traffic light" color-coding, and a faces scale 
Table 1 Eligibility criteria

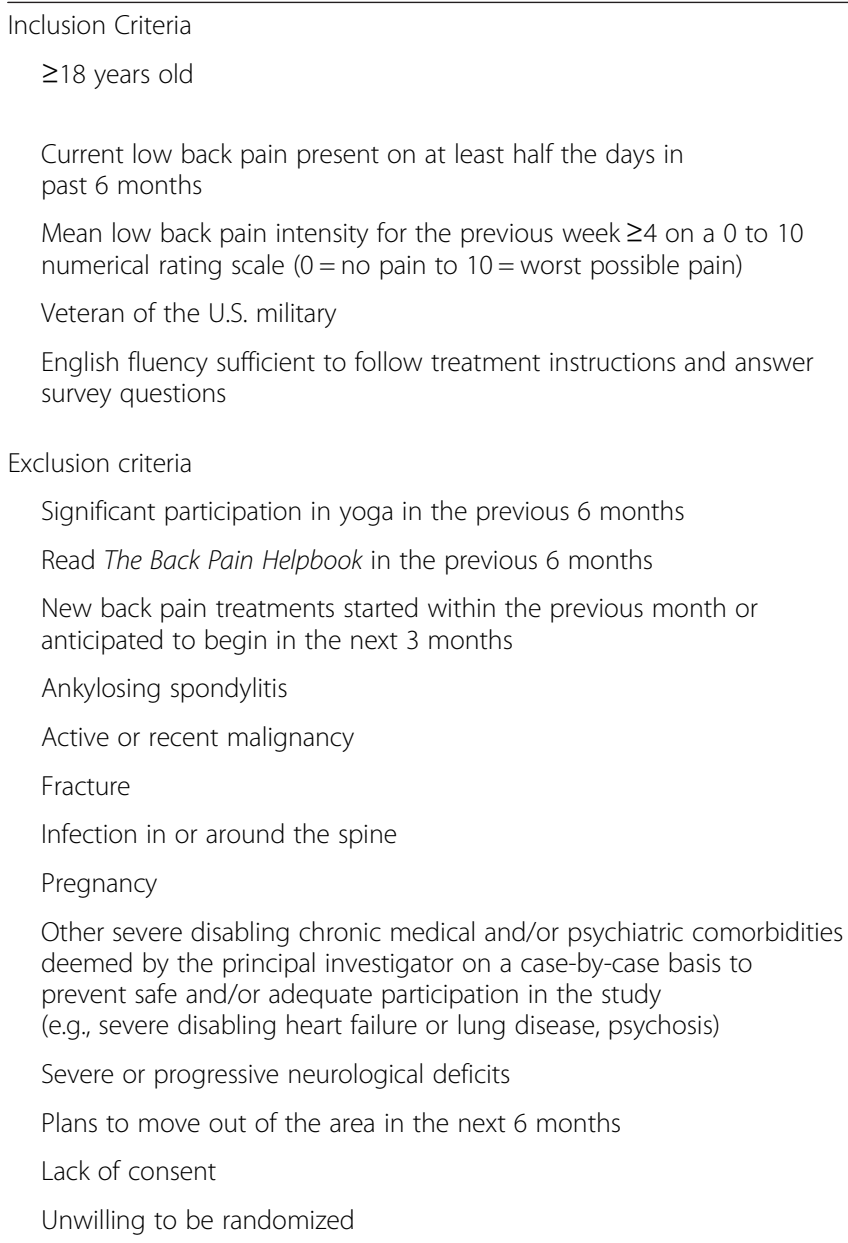

unwillingness to be randomized; or any severe medical or psychiatric condition that, in the principal investigator's judgment, would make participation not possible or unsafe.

\section{Recruitment, enrollment, and retention}

A multipronged strategy for recruitment is being used:

1. Designate "study champions" within different Bedford VA Hospital clinical areas. The champion publicizes and encourages recruitment within their area through presentations to clinicians and staff during regularly scheduled meetings. Clinical site champions ensure all relevant clinicians and staff are aware of the study and provide flyers with information on the study to Veterans when discussing treatment options for pain at the time of encounter/appointment.

2. Identify in the electronic health records Veterans seen in the previous two years at the Bedford VA Hospital who have back pain on their problem list. Targeted letters signed by clinical site champions,

\section{Rationale}

Chronic low back pain in children results from different causes than those we are studying

Condition studied is specifically chronic

Back pain severe enough to detect improvement and prevent against floor effects

Defined target population

Fully informed consent and data collection

Rationale

Possible bias due to current or recent intervention users

Back pain due to, or possibly result of, specific disease/condition(s)

Comorbid condition(s) pose inappropriate risk to safety or preclude compliance with interventions

Known barrier to full study participation

Research policy

flyers, and opt-out cards are mailed to these Veterans inviting them to join the study.

3. Place study flyers and brochures in exam rooms, waiting rooms, and public areas throughout the Bedford VA Hospital and surrounding community.

4. Use Veteran networks established by coinvestigators and stakeholder organizations to advertise the study.

The screening and enrollment process involves (1) eligibility screening and (2) an in-person consent meeting with study staff. Screening for eligibility takes place by telephone using a 12-item questionnaire and is preceded by verbal consent. If the Veteran appears to be eligible based on the telephone screening, he or she is asked to meet in person with research staff for a 30-minute informational meeting. The meeting includes a detailed discussion of study procedures, interventions, risks and benefits of participation, confidentiality, and expectations of participants. For those interested in joining the study, informed consent is obtained both verbally and in writing. 
Research staff employ a Plan-Do-Study-Act quality improvement process [78] on a weekly basis to continuously monitor and improve recruitment and retention. Recruitment statistics for the previous week and studyto-date are discussed weekly using a standardized format. Attendance rates for informational consent meetings, yoga classes, and survey visits are also reviewed regularly. Based on these data, the team discusses updates to recruitment and retention strategies if necessary.

\section{Randomization}

The baseline survey is typically completed within 45 days after eligibility screening. If the period since screening exceeds 45 days, eligibility is re-assessed and verified prior to the baseline survey. After completion of the baseline survey, the participant is randomized in a 1:1 ratio to yoga or education using StudyTRAX ${ }^{\mathrm{m}}$ (Macon, GA), a HIPAA-compliant study management software platform. Permuted block randomization with varying block sizes of two or four is used. Stratification is done on three dichotomous variables to assure similar distribution between the two groups:

1. Age ( $\leq 45,>45$ years). Back pain in older Veterans may be structurally different. Furthermore, age may be a confounding variable to the response to yoga.

2. PCL-C score $(<30, \geq 30)$. These cut-offs will help assure equivalent numbers of Veterans with PTSS in both treatment arms in order to compare the effectiveness of the interventions for reducing comorbid PTSS. PTSS may also be an effect modifier for yoga and CLBP.

3. Partnered (yes/no). A partnered Veteran is defined as one who reports having a cohabitating or longterm committed partner/spouse. This helps assure adequate numbers of partnered Veterans in the yoga group available for qualitative interviews.

\section{Study interventions}

The study interventions start within two weeks of randomization to allow adequate time for pre-intervention qualitative interviews. All interventions, related supplies, and transportation to study visits are provided at no cost to participants. Veterans in both treatment arms can continue to see their providers and receive routine care including medication.

\section{Hatha yoga}

The structured and manualized yoga protocol for cLBP was initially developed by an expert panel led by the principal investigator (RBS) in 2007. It was used in a pilot study of 30 predominantly minority low-income civilian participants [40]. The protocol was further refined in 2012 for a yoga dosing study comparing one vs. two yoga classes per week [37]. This protocol was again adapted for use in a large study of 320 civilians that compared yoga, physical therapy, and education [79]. In order to adapt the yoga protocol to a Veteran population, a panel of 11 experts and stakeholders convened in November 2014. Attendees included Veterans, military leaders, yoga teachers, representatives from organizations focused on providing mind-body therapies to Veterans (There \& Back Again $^{\odot}$, Warriors at Ease $^{\odot}$ ), and researchers with expertise in yoga, low back pain, and the VHA. Prior to the meeting, relevant materials (previous yoga for back pain protocols and literature) were distributed for the attendees to review. The fullday in-person meeting included presentations and discussions of unique aspects to teaching yoga to Veterans, class format, segment themes, relaxation exercises, breathing exercises, and specific postures. Based on this input, iterative drafts of the yoga instructor documents were revised and circulated until broad consensus was obtained on the final version.

Yoga classes contain no more than eight to ten Veterans and two yoga teachers to assure a low Veteran-toinstructor ratio (no more than five Veterans to one teacher). This arrangement maximizes effectiveness and safety, and allows flexibility in yoga teacher scheduling based on the study's needs. Veterans are asked to attend one 75-minute class per week and receive reminder phone calls the day before each scheduled class. Attendance is taken by research staff. We provide $\$ 10$ travel compensation for each yoga session. Mats and props are provided. Each class includes a yoga breathing exercise (pranayama), discussion of yoga philosophical principles, yoga postures (asanas), and a relaxation exercise (svasana). Veterans are frequently advised to proceed carefully and slowly. The difficulty of postures increases throughout the 12 weeks (Table 2). The 12-week intervention period is divided into four 3-week segments, titled Opening to Something New, Listening to Your Back, Engaging Your Power, and Bringing it Home. Posture variations, modifications, and various aids (e.g., block, chair, strap, wall) are built into the protocol to accommodate a range of abilities. Yoga participants are encouraged to practice at home for 30 minutes on days when they do not have class. To help Veterans practice at home, they receive a guidebook describing and depicting the protocol (see Additional file 1), yoga mat, strap, two blocks, and home practice instructional videos (see Additional file 2-5). Home practice videos portray Veterans in an introductory video describing the value of yoga practice in their own lives, two 30-minute videos that follow the same structure as group yoga classes, and one 20-minute extended breathing and relaxation video. Veterans are given a link to view the home practice videos online or, if requested, they receive a take-home DVD. 
Table 2 Twelve-week standardized hatha yoga protocol

\begin{tabular}{|c|c|c|c|c|}
\hline \multirow[t]{4}{*}{ Yoga posture (asana) or class component } & \multicolumn{4}{|c|}{ Classes incorporating component by segment } \\
\hline & Segment 1 & Segment 2 & Segment 3 & Segment 4 \\
\hline & Weeks 1-3 & Weeks 4-6 & Weeks 7-9 & Weeks 10-12 \\
\hline & Opening to Something New & Listening to Your Back & Engaging Your Power & Bringing it Home \\
\hline Breathing exercise & $\checkmark$ & $\checkmark$ & $\checkmark$ & $\checkmark$ \\
\hline Knees to chest pose ${ }^{*}$ & $\checkmark$ & $\checkmark$ & $\checkmark$ & $\checkmark$ \\
\hline Knees together twist pose & $\checkmark$ & $\checkmark$ & $\checkmark$ & $\checkmark$ \\
\hline Pelvic tilt pose ${ }^{*}$ & $\checkmark$ & & & \\
\hline Big toe pose ${ }^{*}$ & & $\checkmark$ & $\checkmark$ & $\checkmark$ \\
\hline Cat and cow pose* & $\checkmark$ & & $\checkmark$ & \\
\hline Wheel pose ${ }^{*}$ & $\checkmark$ & & $\checkmark$ & \\
\hline Chair pose $^{*}$ & $\checkmark$ & $\checkmark$ & $\checkmark$ & \\
\hline Shoulder openers ${ }^{*}$ & $\checkmark$ & $\checkmark$ & $\checkmark$ & $\checkmark$ \\
\hline Crescent moon pose ${ }^{*}$ & $\checkmark$ & $\checkmark$ & & $\checkmark$ \\
\hline Mountain pose ${ }^{*}$ & $\checkmark$ & $\checkmark$ & $\checkmark$ & $\checkmark$ \\
\hline Child's pose* & $\checkmark$ & $\checkmark$ & $\checkmark$ & $\checkmark$ \\
\hline Locust pose & $\checkmark$ & $\checkmark$ & $\checkmark$ & \\
\hline Sphinx pose ${ }^{*}$ & $\checkmark$ & $\checkmark$ & & \\
\hline Cobra pose $^{*}$ & & $\checkmark$ & & $\checkmark$ \\
\hline Plank pose* & & & $\checkmark$ & $\checkmark$ \\
\hline Side plank pose & & & $\checkmark$ & $\checkmark$ \\
\hline Downward facing dog ${ }^{*}$ & $\checkmark$ & $\checkmark$ & $\checkmark$ & $\checkmark$ \\
\hline Triangle pose $^{*}$ (with and without wall) & $\checkmark$ & $\checkmark$ & $\checkmark$ & $\checkmark$ \\
\hline Forward bend pose (with and without wall) & $\checkmark$ & & & $\checkmark$ \\
\hline Warrior I pose ${ }^{*}$ & $\checkmark$ & & $\checkmark$ & $\checkmark$ \\
\hline Warrior II pose ${ }^{*}$ & & $\checkmark$ & $\checkmark$ & $\checkmark$ \\
\hline Wide-leg bend pose (with and without wall) & & $\checkmark$ & $\checkmark$ & \\
\hline Side hip strengtheners & & $\checkmark$ & $\checkmark$ & $\checkmark$ \\
\hline Eye of the needle pose ${ }^{*}$ & & $\checkmark$ & $\checkmark$ & $\checkmark$ \\
\hline Extended leg pose* & & $\checkmark$ & & $\checkmark$ \\
\hline Baby dancer pose ${ }^{*}$ (modified at the wall) & & & & $\checkmark$ \\
\hline Bridge pose (with and without support) & $\checkmark$ & $\checkmark$ & $\checkmark$ & $\checkmark$ \\
\hline Reclining cobbler pose & & $\checkmark$ & $\checkmark$ & $\checkmark$ \\
\hline Reclining chest opener pose & & $\checkmark$ & $\checkmark$ & $\checkmark$ \\
\hline Legs up the wall pose & & & $\checkmark$ & $\checkmark$ \\
\hline Relaxation exercise & $\checkmark$ & $\checkmark$ & $\checkmark$ & $\checkmark$ \\
\hline
\end{tabular}

*Pose has optional chair modifications described in teacher and participant manuals

All yoga teachers underwent training prior to teaching study classes. A 6-hour in-person training covered the specific yoga practices suggested (e.g., postures, breathing exercises) and information on how to teach a culturally-sensitive yoga class to Veterans. Online webinars provided by Warriors at Ease ${ }^{\odot}$ included four 90 minute courses on cultural sensitivity, common injuries or conditions in Veterans, the role of the yoga teacher(s), and managing emotional reactions. All teachers received and follow an instructor manual (see Additional file 6), which contains detailed information on the segment themes, week-by-week yoga class structure, teaching individual postures with or without modifications, leading breathing exercises, and guiding Veterans through relaxation. To assess fidelity to the protocol, researchers observe $10 \%$ of classes using a protocol fidelity checklist. 


\section{Education}

The Back Pain Helpbook [68] is given to individuals randomized to the education group. This book has been used successfully in previous cLBP studies for educational purposes [32, 80]. The book includes information on how back pain is influenced by posture, emotions, fear avoidance behaviors, and social support. It also encourages pain self-management strategies such as mind-body relaxation techniques, exercise programs, and medications. In addition, Veterans receive a handout after randomization suggesting specific chapters to read throughout the intervention period and brief mailed newsletters every three weeks that highlight main points from the recommended chapters (see Additional file 7). Check-in calls, which are proceeded by a newsletter mailing, are conducted by research staff every three weeks to assess progress and reinforce study retention efforts.

\section{Data collection}

Outcome data are collected at baseline, 6 weeks, 12 weeks, and 24 weeks. Participants in both treatment arms complete paper surveys in person and receive cash honoraria after the completion of each survey. We give $\$ 25$ at baseline, 6 weeks, and 12 weeks. At the final follow-up at 24 weeks, participants receive $\$ 50$ after completion of their survey. Paper surveys are doubleentered into StudyTRAX ${ }^{\text {Tu }}$ by research staff who have no knowledge of or access to identifiable participant information or treatment assignment. The two data entries are compared and reconciled.

\section{Outcome measures}

Table 3 shows the data collection schedule. Our measures include core patient-oriented outcomes for the cLBP trials, including those recommended by the Report of the NIH Task Force on Research Standards for Chronic Low Back Pain [73]. Change in pain intensity and back-related function are the two co-primary outcomes at 12 weeks $[63,71]$. Secondary back pain outcomes include pain medicine use in the previous week, including medication class and dosage [81]; health-related quality of life (SF-12) [82]; pain interference [83]; overall improvement (7-point Likert scale, $0=$ extremely worsened to $6=$ extremely improved); and patient satisfaction with CLBP treatment (5point Likert scale, $1=$ very satisfied to $5=$ very dissatisfied) [84]. Psychological secondary outcomes include posttraumatic stress symptoms (PCL-C) [85] and depression symptoms measured using the 9-item Patient Health Questionnaire (PHQ-9) [86]. The Pittsburgh Sleep Quality Index (PSQI) is also administered [87]. Exploratory neuropsychological outcomes include post-concussive symptoms measured using the Neurobehavioral Symptom Inventory (NSI) [88], 7-item Generalized Anxiety Disorder questionnaire (GAD-7) [89], Coping Strategies Questionnaire (CSQ)
[90], and Pain Self-Efficacy Questionnaire (PSEQ) [91]. Exploratory social outcomes include marital/family functioning measured using the Dyadic Adjustment Scale (DAS) [92], employment status (Work Productivity and Activity Impairment questionnaire) [93], and housing status. NIH PROMIS $^{\circ}$ (Patient Reported Outcomes Measurement Information System) tools [94] for outcomes of interest, such as pain, function, depression, anxiety, and sleep, are used to assess how well they correlate with the DVPRS, RMDQ, PHQ-9, GAD-7, and PSQI. At baseline, we also gather standard sociodemographic information and possible covariates, including race, ethnicity, income, education, military service history, medical comorbidities, back pain history, expectation of helpfulness for the different interventions [95], and preference of treatment assignment.

\section{Cost-effectiveness}

We use a multi-method approach to collect cost data at baseline, 6 weeks, 12 weeks, and 24 weeks. Since our interventions may influence other common comorbidities of cLBP (e.g., depression [52]), we measure both backrelated utilization and total medical utilization. Direct medical costs are measured and consist of the cost of (1) implementing the interventions themselves and (2) ongoing medical utilization before, during, and after the intervention. Intervention implementation costs (e.g., non-study-specific staff hours, materials, facility use) are captured from study records and valued at their actual costs. Ongoing total VA medical utilization including visits, hospitalizations, tests, radiology, and medications are taken directly from the electronic medical record system. Direct medical costs are valued at their actual costs to the VA. Any non-VA medical utilization (e.g., chiropractors, out-of-pocket back-related expenses) is obtained from a cost questionnaire completed by the Veteran with the primary initial recall period being the past 6 weeks and is valued at the reported actual price paid by Veterans. Indirect costs (i.e., lost productivity) for employed Veterans will be calculated as the number of lost productive hours (as reported in the Work Productivity and Activity Impairment questionnaire) multiplied by a national average cost of employment for each Veteran's general job category. Lost productivity costs for those not in the work force are assumed to be captured by their report of overall quality of life [96]. Lost productivity costs for those looking for work will be considered in the sensitivity analyses.

\section{Qualitative interviews}

Qualitative interviews occur pre-intervention and postintervention with a subset of partnered yoga participants to explore the impact of cLBP and comorbid mental health disorders on family and marital processes. This will also provide a greater detailed understanding of yoga's perceived impact of on the Veteran and possibly the 
Table 3 Schedule of assessments

\begin{tabular}{|c|c|c|c|c|c|}
\hline Measures & Enrollment & Baseline & 6 weeks & 12 weeks & 24 weeks \\
\hline \multicolumn{6}{|l|}{ Screening and enrollment } \\
\hline Eligibility screening & $\checkmark$ & & & & \\
\hline Informed consent & $\checkmark$ & & & & \\
\hline \multicolumn{6}{|l|}{ Baseline information } \\
\hline Sociodemographics* & & $\checkmark$ & & & \\
\hline Expectations and preference & & $\checkmark$ & & & \\
\hline Back pain history and comorbidities & & $\checkmark$ & & & \\
\hline \multicolumn{6}{|l|}{ Primary outcomes } \\
\hline Low back pain intensity (DVPRS) & & $\checkmark$ & $\checkmark$ & $\checkmark$ & $\checkmark$ \\
\hline Back-related function (RMDQ) & & $\checkmark$ & $\checkmark$ & $\checkmark$ & $\checkmark$ \\
\hline \multicolumn{6}{|l|}{ Secondary outcomes } \\
\hline Pain medication use & & $\checkmark$ & $\checkmark$ & $\checkmark$ & $\checkmark$ \\
\hline PTSD CheckList_Civilian version (PCL-C) & & $\checkmark$ & $\checkmark$ & $\checkmark$ & $\checkmark$ \\
\hline Health-related quality of life (SF-12) & & $\checkmark$ & $\checkmark$ & $\checkmark$ & $\checkmark$ \\
\hline Satisfaction with treatment & & $\checkmark$ & $\checkmark$ & $\checkmark$ & $\checkmark$ \\
\hline Global improvement & & & $\checkmark$ & $\checkmark$ & $\checkmark$ \\
\hline \multicolumn{6}{|l|}{ Cost-effectiveness outcomes } \\
\hline Work productivity & & $\checkmark$ & $\checkmark$ & $\checkmark$ & $\checkmark$ \\
\hline Medical utilization and cost & & $\checkmark$ & $\checkmark$ & $\checkmark$ & $\checkmark$ \\
\hline \multicolumn{6}{|l|}{ Exploratory outcomes } \\
\hline PROMIS-29 and pain interference & & $\checkmark$ & $\checkmark$ & $\checkmark$ & $\checkmark$ \\
\hline Depression (PHQ-9) and anxiety (GAD-7) & & $\checkmark$ & $\checkmark$ & $\checkmark$ & $\checkmark$ \\
\hline Relationship satisfaction (DAS) & & $\checkmark$ & & $\checkmark$ & $\checkmark$ \\
\hline Pain self-efficacy (PSEQ) & & $\checkmark$ & & $\checkmark$ & $\checkmark$ \\
\hline Sleep quality (PSQI) & & $\checkmark$ & & $\checkmark$ & $\checkmark$ \\
\hline Coping strategies (CSQ) & & $\checkmark$ & & $\checkmark$ & $\checkmark$ \\
\hline Post-concussive symptoms (NSI) & & $\checkmark$ & & $\checkmark$ & $\checkmark$ \\
\hline \multicolumn{6}{|l|}{ Possible covariates and confounders } \\
\hline Exercise history & & $\checkmark$ & $\checkmark$ & $\checkmark$ & $\checkmark$ \\
\hline Low back pain treatments & & $\checkmark$ & $\checkmark$ & $\checkmark$ & $\checkmark$ \\
\hline Alcohol, drugs, and smoking & & $\checkmark$ & & $\checkmark$ & $\checkmark$ \\
\hline
\end{tabular}

*Sociodemographic information includes gender, age, relationship status, ethnicity, race, income, housing, education level, and military service history DVPRS = Defense and Veterans Pain Rating Scale; RMDQ = Roland Morris Disability Questionnaire; PROMIS = Patient Reported Outcome Measurement Information System; SF-12 = Short Form 12-item Health Survey; PHQ-9 = 9-item Patient Health Questionnaire; GAD-7 = 7-item General Anxiety Disorder survey; DAS = Dyadic Adjustment Scale; PSQI = Pittsburgh Sleep Quality Index; NSI = Neurobehavioral Symptom Inventory

Veteran's family. After randomization, a purposive sample of partnered Veterans $(n=20$ couples) in the yoga arm are recruited to participate. To be eligible for interviews, Veterans must report having a cohabitating or long-term committed partner/spouse. In addition, both the Veteran and the Veteran's partner must give consent to participate in the interviews. Sampling takes place in a logistically feasible way until the goal sample size is reached.

Separate and conjoint interviews are conducted by a trained qualitative interviewer with the Veteran and his or her partner at two time points - prior to participation in yoga and approximately four weeks after ending classes - in order to maximize the opportunity to authentically discuss the impact of cLBP and yoga on family relationships. Interviews are conducted with each member of the couple individually followed by a short conjoint interview. We encourage couples to attend interviews at the same time. However, if this is not possible, we conduct the two interviews within approximately 72 hours. Semistructured interview guides incorporate the following domains: the Veteran's perception of the impact of cLBP on himself or herself and the partner or family in terms of mood and functioning; the partner's perception of the impact of the Veteran's cLBP on the Veteran's mood and functioning; 
the partner's perception of the Veteran's cLBP on her/his own and overall family mood and functioning; the attitude of the Veteran and partner toward yoga; the Veteran and partner's perception of change in the Veteran's cLBP; the Veteran and partner's perception of any possible change in psychological concerns including PTSS, depression, and anxiety after yoga; and the Veteran and partner's perception of any change in overall family mood and functioning after yoga classes (see Additional file 4).

Interviewers obtain informed consent from the Veteran and partner prior to the interviews. Each individual receives $\$ 25$ for each $60-90$ minute interview visit. Interviews take place at a location convenient to the couple.

If a couple completes a pre-intervention interview but declines to complete a second interview, we attempt to replace that couple with another couple.

\section{Adverse events and data monitoring}

The same strategy for collecting adverse event data is implemented for both study arms. Participants are instructed at enrollment to contact research staff immediately if they experience any adverse event during the study. All participants have 24-hour emergency contact information for the site investigator and another member of the research team. All post-baseline surveys and education check-in calls include questions on whether the Veteran believes he or she has incurred any possible adverse events. Veterans in the yoga group are given the opportunity to speak with a research staff member regarding any potential adverse events during attendance and check-ins at yoga classes. Research staff and the principal investigator follow up on all adverse event reports as appropriate. Unanticipated problems (unexpected, related, and serious) are reported to the IRBs and sponsor within 48 hours.

A Data and Safety Monitoring Board (DSMB) reviews the study conduct and results throughout recruitment, data collection, and treatment implementation phases. The DSMB is composed of four members who are not involved in the conduct of the study. The DSMB meets semiannually throughout the study period to review all adverse event reports, accrual progress, and retention. A summary report of the board's findings and recommendations are submitted to the sponsor and IRBs.

This study will be stopped prior to its completion if: (1) the intervention(s) are associated with adverse effects that significantly impact the risk-benefit ratio; (2) study recruitment or retention becomes futile; (3) any new information becomes available during the trial that necessitates stopping the trial; or (4) other situations occur that might warrant stopping the trial.

\section{Data analysis}

We will conduct both intention-to-treat and per-protocol analyses, with the intention-to-treat analysis being primary.
Descriptive statistics including means, medians, and standard deviations for continuous variables, and the number and proportions for categorical variables will be reported. All analyses will be performed at the $\alpha=0.05$ level of significance.

The success of randomization will first be assessed. We will compare the two groups on baseline characteristics and demographics, including stratification variables, with Student's t test or chi-square test, as appropriate, using an $\alpha=0.1$ level of significance. Variables that differ between the groups at baseline will be considered possible confounders and adjusted for in subsequent analyses.

\section{Primary outcome analyses}

The primary hypothesis is that 12 weeks of yoga will be more effective than education for improving pain and function. For both outcomes, we will determine if yoga is superior to education based on a longitudinal model incorporating all measurements across the study period, including baseline, week 6 , week 12 , and week 24 . The primary hypothesis will be tested using a contrast of the change from baseline to week 12 comparing the two groups within this model. To account for correlation among repeated measures for the same individual, we will assume an unstructured covariance for the initial model. A simpler model may be used for the covariance if appropriate. No missing data will be replaced in these analyses, and all available data can be included. The analysis is considered to be unbiased under a missing-atrandom assumption. It is possible that any missing data may be non-ignorably missing rather than missing-atrandom. We will perform sensitivity analyses to determine if the results are robust to the missing data mechanism. Note that we require statistical significance for both outcomes rather than either outcome to address the issue of multiple testing. We will report mean differences between groups with standard errors, $95 \%$ confidence intervals, and $p$ values. If the analysis of the success of randomization finds any imbalance between groups, the above model will be adjusted for these factors.

Per-protocol analysis will also be conducted similarly. Adherence to the yoga protocol will be defined as attending at least 9 of the 12 yoga classes. For education, adherence will be defined as reading at least three-fourths of the book by self-report.

\section{Secondary back pain outcome analyses}

For pain medication use, we will examine the overall use during the previous week at week 12. First, a chi-square test will be used to compare medication use at week 12 between groups. Next, logistic regression with terms for treatment group and adjusting for potential confounders, including baseline pain medication use, will be used to compare medication usage rates. Use of medication 
subtypes, including nonsteroidal anti-inflammatory drugs, acetaminophen, and opioids, will be similarly compared. We will report odds ratios with corresponding $95 \%$ confidence intervals and $p$ values. Physical and mental health summary scores will be calculated from SF-12 data using population normative data and means compared between groups as for the primary outcomes above. We will also compare the proportion of participants in each group achieving $\geq 30 \%$ and $\geq 50 \%$ improvements from baseline for the co-primary outcomes, often considered to correspond to minimal and moderate clinically meaningful change, respectively.

\section{Psychological and exploratory analyses}

Our hypothesis is that yoga will be superior compared to education for reducing PTSS in the subset of participants with comorbid PTSS (baseline PCL-C $\geq 30$ ). We will first compare PCL-C scores between groups for the subset of participants who qualify as having PTSS at baseline using methods similar to those described above for primary outcome analyses. We will also examine the whole study sample and the subset of participants with lower baseline PCL-C scores. We stratify for this variable during randomization and thus anticipate similar numbers and baseline characteristics between the two study groups. We will also explore whether a change in PTSS is an effect modifier for our primary outcomes of pain and function. We will similarly compare yoga to education for the subgroup of participants with moderate or greater depressive symptoms (PHQ-9 210$)$ [97] and those with less depressive symptoms. Exploratory subset analyses will also be conducted for Veterans scoring moderate or higher on anxiety symptoms (GAD-7 $\geq 10)$ and sleep difficulties (PSQI $>5$ ). Similarly, an exploratory subset analysis will be conducted for participants with post-concussive symptoms, defined as scoring $\geq 20$ on the NSI [98].

Since back pain in older Veterans may be different structurally compared to younger Veterans, we will also complete exploratory subset analyses of Veterans $\leq 45$ and $>45$ years old to assess if age confounds the response to yoga.

To analyze intermediate-term outcomes, we will examine changes from baseline to week 6 using methodology similar to the primary 12 -week analyses. Additional subgroup longitudinal analyses can be done using only data from those participants who were 12-week completers. We will also compare the change in pain medication usage over time using a generalized-estimating equation approach or nonlinear mixed effects model to account for the repeated measures of a dichotomous outcome. This approach will parallel the longitudinal analyses for continuous outcomes described above.

\section{Cost-effectiveness analyses}

All cost-effectiveness analyses will use the intention-totreat principle. Quality-adjusted life-years (QALYs) will be calculated from the results of the SF-12 based on an algorithm developed by Brazier et al. [99] For the Veteran perspective, we will compare Veterans' incremental out-of-pocket costs (e.g., transportation, overthe-counter medications, and co-payments) to their incremental QALY impacts. For the VHA perspective, incremental direct intervention costs will be compared to ongoing direct medical utilization costs. This costbenefit analysis will address whether, economically, yoga should be a treatment option for the VA population. For the society perspective, we will conduct a cost-utility analysis by comparing the incremental societal costs for each treatment arm (i.e., direct medical, nonmedical, and productivity costs) to the incremental change in QALYs [100]. We will use bootstrap methods to calculate confidence intervals [101] and perform one-way sensitivity analyses to determine the robustness of our estimates with different assumptions used to value productivity [102]. Combined non-back pain and back pain-related utilization will be used for all of these analyses. We will also perform sensitivity analyses using only back painrelated utilization.

\section{Qualitative analyses}

Qualitative interviews will be downloaded from digital recorders, transcribed, and cleaned by qualitative research staff. Given the exploratory nature of the interviews, codes will be developed both inductively and deductively using techniques from Grounded Theory [103]. Initially, three research team members will read transcripts from three dyads (six interviews) to identify content categories using the process of line-by-line coding. In order to establish reliability, the team will then confer to discuss codes, condense overlapping areas, and consider alternative possibilities with the goal of developing a coding schema to be used with all interviews. Subsequently, two team members will read each of the remaining pairs of transcripts and continue to conduct focused coding using the decidedupon schema. The team will confer every four transcripts to insure that the codes remain relevant and accurate given the research questions. Where necessary, coding categories will be changed and condensed. This process of line-by-line and focused coding will ultimately lead to axial coding, where larger thematic categories and relationships between them are identified. Matrices will be utilized to compare and contrast code categories and individual cases. The software program NVivo $\left(\mathrm{QSR}^{\odot}\right.$, Melbourne, Australia) will be used for qualitative data management and analysis.

\section{Discussion}

The proposed RCT will (1) establish a structured reproducible yoga protocol uniquely suited to Veteran populations with CLBP and associated psychological 
comorbid symptoms; (2) increase our knowledge of the feasibility and impact of yoga on Veterans' cLBP, psychological comorbidities, and family functioning; and (3) provide a strong foundation for larger multisite studies and implementation projects.

The Institute of Medicine report Relieving Pain in America recognized that protocols for pain management approaches must be adapted to the unique needs of Veterans and undergo rigorous testing for clinical effectiveness and cost-effectiveness [3]. Cost analyses have been infrequently applied to complementary and integrative medicine treatments in general $[104,105]$, and to the best of our knowledge and according to the published literature, never for integrative medicine or yoga in a military setting. Group self-care interventions such as yoga, if effective, have the potential to be cost saving to the VHA and cost-effective to society.

Research of yoga as a therapeutic modality is relatively new and even less well developed for military populations. Given the complexity and heterogeneity of yoga practices [106], structured protocols that are evidence-based and reproducible are critical if wide dissemination is to ultimately occur. The design and implementation of our yoga intervention for the unique needs of Veteran populations with CLBP and associated psychological comorbidities is novel. This study adapts an existing structured yoga protocol validated in civilian populations using an expert panel including Veterans and yoga instructors with expertise in teaching Veterans.

The results of this study have the potential to impact the approach and management of cLBP in accordance with the DoD-VA vision, i.e., a more integrative, interdisciplinary focus on active self-care approaches that empower patients to have greater control of their condition [63]. Despite enthusiasm for offering yoga to Veterans, yoga instruction to date is not well standardized or implemented widely. More importantly, there is little strong evidence for yoga's effect on PTSD, depression, or other psychosocial problems. We anticipate this trial will help determine if yoga can become a safe, clinically effective, cost-effective, and scalable nonpharmacologic approach to address the physical and psychosocial dimensions of cLBP in Veterans.

\section{Trial status}

This trial is currently recruiting participants.

\section{Consent}

Written informed consent was obtained from the yoga home practice video actor(s) for publication of this manuscript and accompanying videos. A copy of the written consent is available for review by the Editor-in-Chief of this journal.

\section{Additional files}

Additional file 1: Participant Yoga Manual. A take-home guidebook for yoga participants describing and depicting yoga exercises to aid home practice. (PDF $4.52 \mathrm{mb}$ )

Additional file 2: Yoga Home Practice Introduction Video. An introductory video that presents Veterans describing the value of yoga practice in their own lives and explains how to set up home practice. (MOV $315 \mathrm{mb}$ )

Additional file 3: Yoga Home Practice Video Weeks 1-6. A 30-minute home practice video that parallels yoga classes in weeks $1-6$ of the study. (MOV $912 \mathrm{mb}$ )

Additional file 4: Yoga Home Practice Video Weeks 7-12. A 30-minute home practice video that parallels yoga classes in weeks 7-12 of the study. (MOV $1.33 \mathrm{gb}$ )

Additional file 5: Yoga Breathing and Relaxation Video. A 20-minute video containing extended breathing and relaxation exercises. (MOV $703 \mathrm{mb}$ )

Additional file 6: Yoga Teacher Manual. A training and reference manual given to study yoga instructors that includes detailed information on the segment themes, weekby- week yoga class structure, teaching individual postures with or without modifications, teaching breathing exercises, and leading Veterans through integrated relaxation. (PDF $3.52 \mathrm{mb}$ )

Additional file 7: Manual of Procedures. Comprehensive manual of standard operating procedures for recruitment, enrollment, data collection/management, interventions, qualitative interviews, and adverse events. (PDF $5.66 \mathrm{mb}$ )

\section{Abbreviations}

CLBP: Chronic low back pain; CSQ: Coping Strategies Questionnaire; DAS: Dyadic Adjustment Scale; DVPRS: Defense and Veterans Pain Rating Scale; GAD-7: 7-item General Anxiety Disorder questionnaire; NCCIH: National Center for Complementary and Integrative Health; NSI: Neurobehavioral Symptom Inventory; OEF: Operation Enduring Freedom; OIF/

OND: Operations Iraqi Freedom/New Dawn; PCL-C: PTSD CheckList - Civilian version; PHQ-9: 9-item Patient Health Questionnaire; PROMIS: PatientReported Outcomes Measurement Information System; PSEQ: Pain SelfEfficacy Questionnaire; PSQI: Pittsburgh Sleep Quality Index; PTSD: PostTraumatic Stress Disorder; PTSS: Post-traumatic stress symptoms; QALY: Quality-adjusted life year; RCT: Randomized controlled trial; RMDQ: Roland-Morris Disability Questionnaire; SF-12: Short form 12-item health survey; VA: Department of Veteran Affairs; VHA: Veterans Health Administration.

\section{Competing interests}

The authors declare that they have no competing interests.

\section{Authors' contributions}

RBS conceived the study, participated in its design and coordination, and drafted the manuscript. CML participated in the study design and coordination, development of the study operating procedures, assisted in yoga video production, and drafted the manuscript. ARE participated in the study design and coordination. RP designed the qualitative component of the study. PMH designed the cost effectiveness analysis. DNP participated in the study design and coordination. KJS, EJG, and SW helped with study design and drafting the manuscript. SL scripted yoga home practice videos, participated in the design of the yoga protocol, and contributed to the yoga manuals. JW designed the quantitative data analysis plan and participated in the study design. All authors read and approved the final manuscript.

\section{Authors' information}

RBS is an Associate Professor of Family Medicine at Boston University School of Medicine, Assistant Professor of Epidemiology at Boston University School of Public Health and is Director of Integrative Medicine in the Department of Family Medicine at Boston Medical Center. CML is a research project manager in the Department of Family Medicine at Boston Medical Center. ARE is the Director of the Center for Information Dissemination and Education Resources in the VA Boston Healthcare System and Associate Professor of Health Policy \& Management at Boston University School of 
Public Health. RP is Associate Professor and Director of the Family Therapy Certificate Program at the Boston University School of Social Work and a mixed methods researcher. PMH is a senior behavioral scientist at the RAND Corporation, faculty member of the Pardee RAND Graduate School, and a resource economist. DNP is a research project manager in the Center for Healthcare Organization and Implementation Research at the Bedford VA Medical Center. KJS is a senior investigator at the Group Health Research Institute, epidemiologist, and clinical trialist with a focus on complementary medicine practices for low back pain. EJG is an Assistant Professor and Director of the Health Services Research Center in the Department of Family and Preventive Medicine at University of California San Diego School of Medicine and is an investigator with the VA San Diego Healthcare System. SW is a clinical psychologist and research health scientist at the Bedford VA Medical Center. SL is a Gulf War Veteran, yoga teacher, founder of There \& Back Again ${ }^{\circ}$, and an officer in the Judge Advocate General Corp in the U.S. Army Reserves. JW is Professor of Biostatistics at Boston University School of Public Health and Director of the Master of Science in Clinical Investigation program at Boston University School of Medicine.

\section{Acknowledgements}

The authors wish to thank the panel that participated in adapting our yoga protocol for a military Veteran population (Robin Carnes, Carol Faulkner, Patricia Lillis, Andrea Lucie, Eric Schoomaker, and Audrey Schoomaker), our yoga instructors for their input on the yoga protocol and materials (Michelle Anastasi, Keith Beasley, Sheri Breen, Jennifer Johnston, David Schouela, Karen Speckman, Sue Stevens, Lawrence Sullivan, Michael Thomas, and Whitney Willman), and the research team for their assistance and support (Alexandra Femia and Catherine Tong). We would also like to thank Nick Kaufman and NKP Media for producing the yoga home practice videos, the Veterans who appeared in the yoga videos (Steve Palmer, Paul Bradley, and Stephanie Riddle), and Kathy Hames for designing the study logo. The copyright owner of the yoga home practice videos used in this study agrees to publish the videos in an open access journal.

This publication was made possible by grant number 3R01AT005956-05S1 from the National Center for Complementary and Integrative Health (NCCIH) at the National Institutes of Health. Its contents are solely the responsibility of the authors and do not necessarily represent the official views of $\mathrm{NCCIH}$.

\section{Author details}

'Department of Family Medicine, Boston University School of Medicine and Boston Medical Center, Boston, MA, USA. ${ }^{2}$ Center for Information Dissemination and Education Resources, VA Boston Healthcare System, Boston, MA, USA. ${ }^{3}$ Department of Health Policy and Management, Boston University School of Public Health, Boston, MA, USA. ${ }^{4}$ Boston University School of Social Work, Boston, MA, USA. ${ }^{5}$ RAND Corporation, Santa Monica, CA, USA. ${ }^{6}$ Center for Healthcare Organization and Implementation Research, Edith Nourse Rogers Memorial Veterans Hospital, Bedford, MA, USA. ' Group Health Research Institute, Seattle, WA, USA. ${ }^{8}$ Department of Epidemiology, University of Washington, Seattle, WA, USA. 'VA San Diego Healthcare System, San Diego, CA, USA. ${ }^{10}$ Department of Family and Preventive Medicine, University of California San Diego School of Medicine, San Diego, CA, USA. ${ }^{11}$ There \& Back Again, Inc., Wakefield, MA, USA. ${ }^{12}$ Department of Biostatistics, Boston University School of Public Health, Boston, MA, USA.

\section{Received: 15 March 2016 Accepted: 1 April 2016}

\section{Published online: 29 April 2016}

\section{References}

1. Lew HL, Otis JD, Tun C, Kerns RD, Clark ME, Cifu DX. Prevalence of chronic pain, posttraumatic stress disorder, and persistent postconcussive symptoms in OIF/OEF Veterans: polytrauma clinical triad. J Rehabil Res Dev. 2009;46:697-702.

2. Kerns RD. Transforming pain care in the United States Department of Veterans Affairs: the role of the VA Pain Research Network. Clin J Pain. 2013;29:93-4.

3. Institute of Medicine. Relieving pain in America: a blueprint for transforming prevention, care, education, and research. Washington DC: National Academies Press; 2011.

4. Haskell SG, Brandt CA, Krebs EE, Skanderson M, Kerns RD, Goulet JL. Pain among Veterans of Operations Enduring Freedom and Iraqi Freedom: do women and men differ? Pain Med. 2009;10:1167-73.
5. Kirsch B, Berdine H, Zablotsky D, et al. Implementation of a pain management strategy: identifying pain as the fifth vital sign. Vet Health Syst J. 2000:49-59

6. Seal KH, Cohen G, Waldrop A, Cohen BE, Maguen S, Ren L. Substance use disorders in Iraq and Afghanistan Veterans in VA healthcare, 2001-2010: implications for screening, diagnosis and treatment. Drug Alcohol Depend. 2011;116:93-101.

7. Barlas FM, Higgins WB, Pflieger JC, Diecker K. 2011 Department of Defense health related behaviors survey of active duty military personnel. Fairfax: ICF International; 2013.

8. Guo HR, Tanaka S, Halperin WE, Cameron LL. Back pain prevalence in US industry and estimates of lost workdays. Am J Public Health. 1999;89:1029-35.

9. Burstrom K, Johannesson M, Diderichsen F. Swedish population healthrelated quality of life results using the EQ-5D. Qual Life Res. 2001;10:621-35.

10. Kosinski MR, Schein JR, Vallow SM, Ascher S, Harte C, Shikiar R, et al. An observational study of health-related quality of life and pain outcomes in chronic low back pain patients treated with fentanyl transdermal system. Curr Med Res Opin. 2005;21:849-62.

11. Lincoln AE, Smith GS, Amoroso PJ, Bell NS. The natural history and risk factors of musculoskeletal conditions resulting in disability among US Army personnel. Work. 2002;18:99-113.

12. Galloway KT, Buckenmaier III CC, Polomano RC. Special report - war on pain: understanding pain and pain mechanisms. Am Nurse Today. 2011;6(9):3-7.

13. Otis JD, Keane TM, Kerns RD. An examination of the relationship between chronic pain and post-traumatic stress disorder. J Rehabil Res Dev. 2003;40:397-405.

14. McWilliams LA, Cox BJ, Enns MW. Mood and anxiety disorders associated with chronic pain: an examination in a nationally representative sample. Pain. 2003;106:127-33.

15. Haskell SG, Ning Y, Krebs E, Goulet J, Mattocks K, Kerns R, et al. Prevalence of painful musculoskeletal conditions in female and male Veterans in 7 years after return from deployment in Operation Enduring Freedom/ Operation Iraqi Freedom. Clin J Pain. 2012;28:163-7.

16. Jensen MP, Romano JM, Turner JA, Good AB, Wald LH. Patient beliefs predict patient functioning: further support for a cognitive-behavioural model of chronic pain. Pain. 1999;81:95-104.

17. Waddell G, Newton M, Henderson I, Somerville D, Main CJ. A FearAvoidance Beliefs Questionnaire (FABQ) and the role of fear-avoidance beliefs in chronic low back pain and disability. Pain. 1993;52:157-68.

18. Morasco BJ, Corson K, Turk DC, Dobscha SK. Association between substance use disorder status and pain-related function following 12 months of treatment in primary care patients with musculoskeletal pain. J Pain. 2011;12:352-9.

19. Nampiaparampil DE. Prevalence of chronic pain after traumatic brain injury: a systematic review. JAMA. 2008;300:711-9.

20. Kar N. Cognitive behavioral therapy for the treatment of post-traumatic stress disorder: a review. Neuropsychiatr Dis Treat. 2011;7:167-81.

21. Institute of Medicine. Returning home from Iraq and Afghanistan: assessment of readjustment needs of Veterans, service members, and their families. Washington DC: National Academies Press; 2013.

22. Campbell P, Jordan KP, Dunn KM. The role of relationship quality and perceived partner responses with pain and disability in those with back pain. Pain Med. 2012;13:204-14.

23. Leonard MT, Cano A, Johansen AB. Chronic pain in a couples context: a review and integration of theoretical models and empirical evidence. J Pain. 2006; 7:377-90.

24. MacGregor AJ, Corson KS, Larson GE, Shaffer RA, Dougherty AL, Galarneau $M R$, et al. Injury-specific predictors of posttraumatic stress disorder. Injury. 2009:40:1004-10.

25. Koren D, Norman D, Cohen A, Berman J, Klein EM. Increased PTSD risk with combat-related injury: a matched comparison study of injured and uninjured soldiers experiencing the same combat events. Am J Psychiatry. 2005;162:276-82.

26. Paris R, DeVoe ER, Ross AM, Acker ML. When a parent goes to war: effects of parental deployment on very young children and implications for intervention. Am J Orthopsychiatry. 2010;80:610-8.

27. Cozza SJ, Guimond JM, McKibben JB, Chun RS, Arata-Maiers TL, Schneider B, et al. Combat-injured service members and their families: the relationship of child distress and spouse-perceived family distress and disruption. J Trauma Stress. 2010;23:112-5.

28. Saper RB, Eisenberg DM, Davis RB, Culpepper L, Phillips RS. Prevalence and patterns of adult yoga use in the United States: results of a national survey. Altern Ther Health Med. 2004;10:44-9. 
29. Peregoy JA, Clarke TC, Jones LI, Stussman BJ, Nahin RL. Regional variation in use of complementary health approaches by U.S. adults. NCHS Data Brief. 2014; 146:1-8.

30. Birdee GS, Legedza AT, Saper RB, Bertisch SM, Eisenberg DM, Phillips RS. Characteristics of yoga users: results of a national survey. J Gen Intern Med. 2008;23:1653-8.

31. Barnes PM, Bloom B, Nahin RL. Complementary and alternative medicine use among adults and children: United States, 2007. Nat Health Stat Report. 2008;12:1-23.

32. Sherman KJ, Cherkin DC, Erro J, Miglioretti DL, Deyo RA. Comparing yoga, exercise, and a self-care book for chronic low back pain: a randomized, controlled trial. Ann Intern Med. 2005;143:849-56.

33. Chou R, Huffman LH. Nonpharmacologic therapies for acute and chronic low back pain: a review of the evidence for an American Pain Society/American College of Physicians clinical practice guideline. Ann Intern Med. 2007;147:492-504.

34. Williams K, Abildso C, Steinberg L, Doyle E, Epstein B, Smith D, et al. Evaluation of the effectiveness and efficacy of lyengar yoga therapy on chronic low back pain. Spine. 2009;34:2066-76

35. Sherman KJ, Cherkin DC, Wellman RD, Cook AJ, Hawkes RJ, Delaney K, et al. A randomized trial comparing yoga, stretching, and a self-care book for chronic low back pain. Arch Intern Med. 2011;171:2019-26.

36. Tilbrook HE, Cox H, Hewitt CE, Kang'ombe AR, Chuang LH, Jayakody S, et al. Yoga for chronic low back pain: a randomized trial. Ann Intern Med. 2011;155(9):569-78.

37. Saper RB, Boah AR, Keosaian J, Cerrada C, Weinberg J, Sherman KJ. Comparing once- versus twice-weekly yoga classes for chronic low back pain in predominantly low income minorities: a randomized dosing trial. Evid Based Complement Alternat Med. 2013;2013:658030. doi:10.1155/2013/658030.

38. Galantino ML, Bzdewka TM, Eissler-Russo JL, Holbrook ML, Mogck EP, Geigle $P$, et al. The impact of modified Hatha yoga on chronic low back pain: a pilot study. Altern Ther Health Med. 2004;10:56-9.

39. Williams KA, Petronis J, Smith D, Goodrich D, Wu J, Ravi N, et al. Effect of lyengar yoga therapy for chronic low back pain. Pain. 2005;115:107-17.

40. Saper RB, Sherman KJ, Cullum-Dugan D, Davis RB, Phillips RS, Culpepper L. Yoga for chronic low back pain in a predominantly minority population: a pilot randomized controlled trial. Altern Ther Health Med. 2009;15:18-27.

41. Cox H, Tilbrook H, Aplin J, Semlyen A, Torgerson D, Trewhela A, et al. A randomised controlled trial of yoga for the treatment of chronic low back pain: results of a pilot study. Complement Ther Clin Pract. 2010;16:187-93.

42. Tekur $P$, Nagarathna $R$, Chametcha S, Hankey A, Nagendra HR. A comprehensive yoga programs improves pain, anxiety and depression in chronic low back pain patients more than exercise: an RCT. Complement Ther Med. 2012;20:107-18.

43. Nambi GS, Inbasekaran D, Khuman R, Devi S, Shanmugananth, Jagannathan K. Changes in pain intensity and health related quality of life with lyengar yoga in nonspecific chronic low back pain: a randomized controlled study. Int J Yoga. 2014;7:48-53.

44. Posadzki P, Ernst E. Yoga for low back pain: a systematic review of randomized clinical trials. Clin Rheumatol. 2011:30:1257-62.

45. Cramer H, Lauche R, Haller H, Dobos G. A systematic review and metaanalysis of yoga for low back pain. Clin J Pain. 2013;29:450-60.

46. Coeytaux RR, McDuffie J, Goode A, Cassel S, Porter WD, Sharma P, et al. Evidence map of yoga for high-impact conditions affecting Veterans. Washington, DC: Department of Veterans Affairs; 2014.

47. Sherman KJ, Wellman RD, Cook AJ, Cherkin DC, Ceballos RM. Mediators of yoga and stretching for chronic low back pain. Evid Based Complement Alternat Med. 2013;2013:130818. doi:10.1155/2013/130818.

48. Balasubramaniam M, Telles S, Doraiswamy PM. Yoga on our minds: a systematic review of yoga for neuropsychiatric disorders. Front Psychiatry. 2012;3:117.

49. Janakiramaiah N, Gangadhar BN, Naga Venkatesha Murthy PJ, Harish MG, Subbakrishna DK, Vedamurthachar A. Antidepressant efficacy of Sudarshan Kriya Yoga (SKY) in melancholia: a randomized comparison with electroconvulsive therapy (ECT) and imipramine. J Affect Disord. 2000;57:255-9.

50. Kinser PA, Bourguignon C, Whaley D, Hauenstein E, Taylor AG. Feasibility, acceptability, and effects of gentle hatha yoga for women with major depression: findings from a randomized controlled mixed-methods study. Arch Psychiatr Nurs. 2013;27:137-47.

51. Michalsen A, Jeitler M, Brunnhuber S, Ludtke R, Bussing A, Musial F, et al. lyengar yoga for distressed women: a 3-armed randomized controlled trial.
Evid Based Complement Alternat Med. 2012;2012:408727. doi:10.1155/2012/ 408727.

52. Pilkington K, Kirkwood G, Rampes H, Richardson J. Yoga for depression: the research evidence. J Affect Disord. 2005;89:13-24.

53. Saeed SA, Antonacci DJ, Bloch RM. Exercise, yoga, and meditation for depressive and anxiety disorders. Am Fam Physician. 2010;81:981-6.

54. Uebelacker LA, Epstein-Lubow G, Gaudiano BA, Tremont G, Battle CL, Miller IW. Hatha yoga for depression: critical review of the evidence for efficacy, plausible mechanisms of action, and directions for future research. Psychiatr Pract. 2010;16:22-33.

55. Woolery A, Myers H, Sternlieb B, Zeltzer L. A yoga intervention for young adults with elevated symptoms of depression. Altern Ther Health Med. 2004:10:60-3.

56. Streeter CC, Whitfield TH, Owen L, Rein T, Karri SK, Yakhkind A, et al. Effects of yoga versus walking on mood, anxiety, and brain GABA levels: a randomized controlled MRS study. J Altern Complement Med. 2010;16:1145-52.

57. Kohn M, Persson LU, Bryngelsson IL, Anderzen-Carlsson A, Westerdahl E. Medical yoga for patients with stress-related symptoms and diagnoses in primary health care: a randomized controlled trial. Evid Based Complement Alternat Med. 2013;2013:215348. doi:10.1155/2013/215348.

58. Michalsen A, Grossman P, Acil A, Langhorst J, Ludtke R, Esch T, et al. Rapid stress reduction and anxiolysis among distressed women as a consequence of a three-month intensive yoga program. Med Sci Monit. 2005;11:CR555-61.

59. Vollestad J, Nielsen MB, Nielsen GH. Mindfulness- and acceptance-based interventions for anxiety disorders: a systematic review and meta-analysis. $\mathrm{Br}$ J Clin Psychol. 2012;51:239-60.

60. Afonso RF, Hachul H, Kozasa EH, de Souza OD, Goto V, Rodrigues D, et al. Yoga decreases insomnia in postmenopausal women: a randomized clinical trial. Menopause. 2012;19(2):186-93.

61. Khalsa SB. Treatment of chronic insomnia with yoga: a preliminary study with sleep-wake diaries. Appl Psychophysiol Biofeedback. 2004;29:269-78.

62. Evans S, Moieni M, Subramanian S, Tsao JC, Sternlieb B, Zeltzer LK. "Now I see a brighter day": expectations and perceived benefits of an lyengar yoga intervention for young patients with rheumatoid arthritis. J Yoga Phys Ther. 2011;1:101.

63. Office of The Army Surgeon General Pain Management Task Force. Providing a standardized DoD and VHA vision and approach to pain management to optimize the care for warriors and their families. Washington, DC: Office of the Army Surgeon General; 2010.

64. Libby DJ, Reddy F, Pilver CE, Desai RA. The use of yoga in specialized VA PTSD treatment programs. Int J Yoga Therap. 2012;22:79-87.

65. Groessl EJ, Weingart KR, Aschbacher K, Pada L, Baxi S. Yoga for Veterans with chronic low-back pain. J Altern Complement Med. 2008;14:1123-9.

66. Groessl EJ, Weingart KR, Johnson N, Baxi S. The benefits of yoga for women Veterans with chronic low back pain. J Altern Complement Med. 2012:18:832-8.

67. Armed Forces Health Surveillance Center (AFHSC). MSMR. 2013;20:18-23.

68. Moore J, Lorig K, Von Korff M, Gonzalez V, Laurent DD. The back pain helpbook. Boston: Da Capo Press; 1999.

69. Buckenmaier III CC, Galloway KT, Polomano RC, McDuffie M, Kwon N, Gallagher RM. Preliminary validation of the Defense and Veterans Pain Rating Scale (DVPRS) in a military population. Pain Med. 2013;14:110-23

70. Nassif TH, Hull A, Holliday SB, Sullivan P, Sandbrink F. Concurrent validity of the Defense and Veterans Pain Rating Scale in VA outpatients. Pain Med. 2015;16(11):2152-61.

71. Patrick DL, Deyo RA, Atlas SJ, Singer DE, Chapin A, Keller RB. Assessing healthrelated quality of life in patients with sciatica. Spine. 1995;20:1899-908. discussion 1909

72. Roland M, Fairbank J. The Roland-Morris Disability Questionnaire and the Oswestry Disability Questionnaire. Spine. 2000;25:3115-24.

73. Deyo RA, Dworkin SF, Amtmann D, Andersson G, Borenstein D, Carragee E, et al. Report of the NIH Task Force on Research Standards for Chronic Low Back Pain. Spine J. 2014;39:1128-43.

74. Grotle M, Brox الـ Vollestad NK Concurrent comparison of responsiveness in pain and functional status measurements used for patients with low back pain. Spine. 2004;29:E492-501.

75. Bombardier C, Hayden J, Beaton DE. Minimal clinically important difference. Low back pain: outcome measures. J Rheumatol. 2001:28:431-8.

76. Bormann JE, Smith TL, Becker S, Gershwin M, Pada L, Grudzinski AH, et al. Efficacy of frequent mantram repetition on stress, quality of life, and spiritual well-being in Veterans: a pilot study. J Holist Nurs. 2005;23:395-414. 
77. Polusny MA, Erbes CR, Thuras P, Moran A, Lamberty GJ, Collins RC, et al. Mindfulness-based stress reduction for posttraumatic stress disorder among Veterans: a randomized clinical trial. JAMA. 2015;314:456-65.

78. Berwick DM. Developing and testing changes in delivery of care. Ann Intern Med. 1998;128:651-6.

79. Saper RB, Sherman KJ, Delitto A, Herman PM, Stevans J, Paris R, et al. Yoga vs. physical therapy vs. education for chronic low back pain in predominantly minority populations: study protocol for a randomized controlled trial. Trials. 2014;15:67.

80. Cherkin DC, Eisenberg DM, Sherman KJ, Barlow W, Kaptchuk TJ, Street J, et al. Randomized trial comparing Traditional Chinese Medical acupuncture, therapeutic massage, and self-care education for chronic low back pain. Arch Intern Med. 2001;161:1081-8.

81. Costa LO, Maher CG, Latimer J, Ferreira PH, Ferreira ML, Pozzi GC, et al. Clinimetric testing of three self-report outcome measures for low back pain patients in Brazil: which one is the best? Spine. 2008;33:2459-63.

82. Ware Jr J, Kosinski M, Keller SD. A 12-Item Short-Form Health Survey: construction of scales and preliminary tests of reliability and validity. Med Care. 1996:34:220-33.

83. Bombardier C. Outcome assessments in the evaluation of treatment of spinal disorders: introduction. Spine. 2000;25:3097-9.

84. Hudak PL, Wright JG. The characteristics of patient satisfaction measures. Spine. 2000;25:3167-77.

85. Weathers FW, Litz BT, Huska JA, Keane TM. PTSD Checklist-Civilian version (PCL-C). Boston: National Center for PTSD, Behavioral Science Division; 1991.

86. Lowe B, Kroenke K, Herzog W, Grafe K. Measuring depression outcome with a brief self-report instrument: sensitivity to change of the Patient Health Questionnaire (PHQ-9). J Affect Disord. 2004;81:61-6.

87. Buysse DJ, Reynolds III CF, Monk TH, Berman SR, Kupfer DJ. The Pittsburgh Sleep Quality Index: a new instrument for psychiatric practice and research. Psychiatry Res. 1989;28:193-213.

88. Cicerone KD, Kalmar K. Persistent postconcussion syndrome: the structure of subjective complaints after mild traumatic brain injury. J Head Trauma Rehabil. 1995;10:1-17.

89. Spitzer RL, Kroenke K, Williams JB, Lowe B. A brief measure for assessing generalized anxiety disorder: the GAD-7. Arch Intern Med. 2006;166:1092-7.

90. Jensen MP, Keefe FJ, Lefebvre JC, Romano JM, Turner JA. One- and twoitem measures of pain beliefs and coping strategies. Pain. 2003;104:453-69.

91. Anderson KO, Dowds BN, Pelletz RE, Edwards WT, Peeters-Asdourian C. Development and initial validation of a scale to measure self-efficacy beliefs in patients with chronic pain. Pain. 1995;63:77-84.

92. Spanier GB. Measuring dyadic adjustment: new scales for assessing the quality of marriage and similar dyads. J Marriage Fam. 1976;38:15-28.

93. Amick III BC, Lerner D, Rogers WH, Rooney T, Katz JN. A review of healthrelated work outcome measures and their uses, and recommended measures. Spine. 2000;25:3152-60.

94. Reeve BB, Hays RD, Bjorner JB, Cook KF, Crane PK, Teresi JA, et al. Psychometric evaluation and calibration of health-related quality of life item banks: plans for the Patient-Reported Outcomes Measurement Information System (PROMIS). Med Care. 2007;45:S22-31.

95. Kalauokalani D, Cherkin DC, Sherman KJ, Koepsell TD, Deyo RA. Lessons from a trial of acupuncture and massage for low back pain: patient expectations and treatment effects. Spine. 2001;26:1418-24.

96. Weinstein MC, Siegel JE, Gold MR, Kamlet MS, Russell LB. Recommendations of the Panel on Cost-Effectiveness in Health and Medicine. JAMA. 1996;276:1253-8.

97. Kroenke K, Spitzer RL, Williams JB, Lowe B. The Patient Health Questionnaire Somatic, Anxiety, and Depressive Symptom Scales: a systematic review. Gen Hosp Psychiatry. 2010;32:345-59.

98. King PR, Donnelly KT, Donnelly JP, Dunnam M, Warner G, Kittleson CJ, et al. Psychometric study of the Neurobehavioral Symptom Inventory. J Rehabil Res Dev. 2012;49:879-88.

99. Brazier JE, Roberts J. The estimation of a preference-based measure of health from the SF-12. Med Care. 2004:42:851-9.

100. Drummond MF, O'Brien B, Stoddart GL, Torrance GW. Methods for the economic evaluation of healthcare programmes. 3rd ed. Oxford: Oxford University Press; 2005.

101. Thompson SG, Barber JA. How should cost data in pragmatic randomised trials be analysed? BMJ. 2000;320:1197-200.

102. Briggs A. Economics notes: handling uncertainty in economic evaluation. BMJ. 1999;319:120
103. Charmaz K. Constructing grounded theory. London: SAGE Publishing; 2006.

104. Herman PM, D'Huyvetter K, Mohler MJ. Are health services research methods a match for CAM? Altern Ther Health Med. 2006;12(3):78-83.

105. Herman PM, Poindexter BL, Witt CM, Eisenberg DM. Are complementary therapies and integrative care cost-effective? A systematic review of economic evaluations. BMJ Open. 2012;2(5):e001046.

106. Sherman KJ. Guidelines for developing yoga interventions for randomized trials. Evid Based Complement Alternat Med. 2012;2012:143271.

\section{Submit your next manuscript to BioMed Central and we will help you at every step:}

- We accept pre-submission inquiries

- Our selector tool helps you to find the most relevant journal

- We provide round the clock customer support

- Convenient online submission

- Thorough peer review

- Inclusion in PubMed and all major indexing services

- Maximum visibility for your research

Submit your manuscript at www.biomedcentral.com/submit
C Biomed Central 